\title{
Effect of dance on multi-muscle synergies in older adults: a cross-sectional study
}

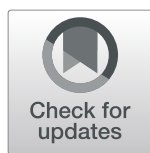

Yun Wang ${ }^{1 *}$, Kazuhiko Watanabe ${ }^{2}$ and Tadayoshi Asaka ${ }^{3}$

\begin{abstract}
Background: The purpose of this study was to investigate the efficacy of dance in the experienced older dancers compared to the inexperienced older adults. We explored the effect of dance on the composition of muscle groups and multi-muscle synergies stabilizing the center of pressure (COP) displacement in preparation to take a step during support surface translation.

Methods: Eight dance experienced elderly participants were asked to take a step in response to support surface perturbations. Uncontrolled manifold analysis was used to identify muscle modes (M-modes) as factors in the muscle activation space. Variance components in the M-mode space and indices of M-mode synergy stabilizing COP displacement were computed.

Results: The reciprocal M-modes were observed more frequently in the dance group than in the control group prior to the step initiation. Dance led to higher indices of multi-muscle synergies and earlier anticipatory synergy adjustments during preparation for making a step in response to the support surface translations.

Conclusions: Dance appeared to be associated with adjustments in both the composition of M-modes and Mmode co-variation patterns resulting in stronger synergies stabilizing COP coordinate in older adults. The results reported here could have clinical relevance when offering a dance approach to balance training for impaired individuals.
\end{abstract}

Keywords: Synergy, Anticipatory adjustments, Perturbation, Dance, Older adults

\section{Background}

Effective maintenance of vertical posture in making a step requires complex control of a moving center of pressure (COP). This task represents a challenge to the postural control system due to a sequence of muscle activations and associated changes in the ground reaction forces serve to unload the stepping leg and propel the body center of mass forward before stepping $[1,2]$. Following a perturbation to stable standing posture, making a step to recover balance is essential for preventing falls and preserving functional mobility. In studies of responses evoked by the anteriorposterior perturbation, older adults were found to be more likely than young adults to initiate stepping at lower levels of instability [3, 4]. Due to age-related neuromuscular decline, increased co-activation of agonist-antagonist muscles

\footnotetext{
* Correspondence: yunwang70@hotmail.com

${ }^{1}$ Tianjin Key Lab of Exercise Physiology and Sports Medicine, College of Social Sport and Health Sciences, Tianjin University of Sport, 16 Donghai Road, Tuanbo Xincheng Xiqu, Jinghai District, Tianjin 301617, China Full list of author information is available at the end of the article
}

and performance decrement [5-7] impede the ability to execute stepping effectively.

A considerable amount of experimental evidence has shown that feed-forward postural control serves to maintain stability by compensating for anticipated destabilization associated with stepping. The central nervous system (CNS) performs anticipatory postural adjustments (APAs) and anticipatory synergy adjustments (ASAs) in the activation patterns of postural muscles before an action or perturbation initiation [8]. APAs typically generate net forces and moments of force counteracting the expected mechanical effects from the anticipated action/perturbation on posture $[9,10]$. In contrast, ASAs attenuate pre-existent synergies in preparation for action in a salient performance variable.

Experimental data has shown changes in indices of synergy with aging [11-13]. In these studies, synergies were defined as co-varied adjustments of independent elemental variables contributing to lower indices of variability of a salient performance variable based on the

(c) The Author(s). 2019 Open Access This article is distributed under the terms of the Creative Commons Attribution 4.0 International License (http://creativecommons.org/licenses/by/4.0/), which permits unrestricted use, distribution, and reproduction in any medium, provided you give appropriate credit to the original author(s) and the source, provide a link to the Creative Commons license, and indicate if changes were made. The Creative Commons Public Domain Dedication waiver (http://creativecommons.org/publicdomain/zero/1.0/) applies to the data made available in this article, unless otherwise stated. 
principle of abundance [14] and the uncontrolled manifold (UCM) hypothesis $[15,16]$. An index of synergy was used to reflect the amount of co-variation among elemental variables that reduced the variability of a task-specific salient performance variable. The UCM approach has been used to investigate the stability of vertical posture across various motor tasks performed by unimpaired and impaired populations $[17,18]$. In this study, groups of coactive muscles with proportional scaling of activation levels were defined as muscle modes (M-modes). Synergy indices that preserve the COP coordinate were quantified using the $\mathrm{M}$-modes as the elemental variables.

A few studies have shown that exercise may lead to a significant improvement in the synergy index in older adults $[19,20]$. In particular, improved finger coordination of older adults was observed in a study of the effects of exercise that challenged performance stability [20]. However, comparatively little is known about the effectiveness of exercise on multi-muscle synergies to maintain or re-establish balance when perturbed. Dance is a complex sensorimotor rhythmic activity that requires dynamic balance and continual adjustment to the environment. Habitual social dancing over several years has shown better balance, gait function, and leg reaction times in older adults [21, 22]. Furthermore, Hackney et al. found that older adults significantly improved their balance and gait when undergoing dance therapy compared with traditional strength/flexibility exercise [23]. Yet how such dance affects the neural control of multimuscle synergies in older adults is not well understood. Therefore, our goal was to determine how the composition of muscle groups and synergies stabilizing the COP coordinate for making a step and balance are affected by dance. Identifying how dance affects links between synergy indices and postural stability in older adults may provide valuable insight into yet unknown mechanisms of the neural control of older adults in anticipation of a perturbation or an action that could guide future rehabilitation efforts.

In the current study, we used support surface translations in the forward direction to explore the effects of dance on multi-muscle postural synergies in the experienced older dancers compared to the inexperienced older adults. Since the muscle activities are dependent on each other $[24,25]$ and redundant, the neural controller forms flexible M-modes by combining the individual muscles to stabilize the COP coordinate. We hypothesized that the composition of muscle groups in the experienced older dancers would be re-organized in preparation for making a step. We predicted that the re-organization of muscle modes with dance would lead to higher posture-stabilizing synergy indices, suggesting greater ability to stabilize the COP coordinate. The confirmation of these hypotheses would inform researchers of the importance of dance in synergic control, and the potential utility of synergy indices for clinical and motor learning studies.

\section{Methods \\ Participant recruitment}

Eight healthy older adults without any neurological, muscular, and/or orthopedic conditions volunteered to participate in the study. Subjects completed a five-year dance class that ran for one and a half hours, twice a week, for approximately 40 weeks a year (allowing for short breaks). Subjects were offered ballroom dancing which included a collection of dances such as Foxtrot, Waltz, and Rumba. Dance programs were not manipulated to mimic exercise training as in aerobic dance classes, nor were they incorporate any specific strength or balance training. Subjects were enrolled by a sample of convenience $(n=11)$ from the entire cohort $(n=20)$. Of these, three subjects who did not obtain a class adherence of at least $80 \%$ during each year of the dance program were excluded. All subjects were right foot dominant. The tests used to determine the dominant foot included kicking a ball, stepping up on a chair, and leaping off in the long jump [26]. To address our primary aim, data from our previous study were used for comparison with the dance group [13]. Ten healthy older adults were recruited from the local community. As the type of physical activity is concerned, we chose eight elderly subjects who walking exercised regularly with no dancing experience as the control group. Table 1 presents the general characteristics and baseline descriptors of the subjects $(p>0.05$ for all). Physical activity level and falls in the past 12 months were assessed by questionnaire. All subjects gave written, informed consent before participation, and the study was approved by the Institutional Review Board of the Tianjin University of Sport. The number of participants was defined based on previous studies of multi-muscle synergies during stepping tasks performed by healthy, young and older adults, which showed moderate-to-large effects of factors such as condition and interval $[13,27]$.

\section{Apparatus}

Surface EMG was recorded in all subjects in the dominant, right lower limb and trunk muscles. Bipolar surface electrodes (approx. $2.5 \mathrm{~cm}$ apart) were placed over the bellies of the following muscles: rectus abdominis (RA), erector spinae (ES), rectus femoris (RF), vastus lateralis (VL), vastus medialis $(\mathrm{VM})$, biceps femoris $(\mathrm{BF})$, semitendinosus (ST), tibialis anterior (TA), lateral head of gastrocnemius (GL), medial head of gastrocnemius (GM), and soleus (SOL). In addition, a reference electrode was attached to the skin over the epicondyle of the tibia. The EMG signals from each muscle were band-pass filtered at $10-500 \mathrm{~Hz}$ and sampled at $1500 \mathrm{~Hz}$ using a Noraxon Telemyo EMG 
Table 1 Subject characteristics and descriptive values

\begin{tabular}{lll}
\hline Variable & Control group $(n=8)$ & Dance group $(n=8)$ \\
\hline Age, $\mathrm{yr}$ & $61.9 \pm 2.9$ & $62.8 \pm 2.6$ \\
Sex, $\mathrm{f} / \mathrm{m}$ & $4 / 4$ & $4 / 4$ \\
Height, $\mathrm{cm}$ & $164.9 \pm 7.7$ & $165.9 \pm 8.2$ \\
Weight, $\mathrm{kg}$ & $66.4 \pm 6.4$ & $66.9 \pm 7.8$ \\
Physical activity & & $5.3 \pm 1.2$ \\
$\quad$ Frequency, d/w & $5.3 \pm 1.8$ & $3.8 \pm 0.8$ \\
$\quad$ Intensity & $3.8 \pm 1.0$ & $8.3 \pm 1.4$ \\
$\quad$ Time of activity, h/w & $8.6 \pm 2.4$ & Dancing \\
$\quad$ Type & Walking & $29.0 \pm 0.9$ \\
Mini mental state examination & $28.9 \pm 0.8$ & 8 \\
\hline falls in the past 12 months, $\mathrm{n}$ & 8 & 8 \\
\hline
\end{tabular}

A scale of 0 to10 for the level of physical exertion was used to assess intensity

Falls was determined by the question: "A fall is when your body goes to the ground without being pushed. Did you fall in the past 12 months?"

system (2400 T V2, Scottsdale, AZ, USA). A force plate (model 9281B, Kistler, Winterthur, Switzerland) captured kinetic data with a frequency of $1500 \mathrm{~Hz}$. The timing of toe-off was measured by a footswitch attached under the heads of the metatarsal bones of the right foot. All subjects were provided with the same thickness socks to wear to secure the sensor firmly in place.

\section{Experimental protocol}

The postural perturbations consisted of forward supportsurface translations with total displacement of $5 \mathrm{~cm}$, peak acceleration of $0.05 \mathrm{~g}$, and peak velocity of $5 \mathrm{~cm} / \mathrm{s}$. Perturbations of this magnitude and speed naturally don't elicit a stepping response when the subjects respond to the perturbation without any instruction [4]. We chose this perturbation based on our observations that the subjects maintained balance without stepping during the support surface translations [27]. Initially, the subject stood on the force plate with equally distributed weight with his/her arms hanging loosely by the sides. The foot position was marked on the force plate and reproduced across trials. The subject was required to take a step forward with his/ her right leg in response to perturbations from quiet stance. The step was made on a flat surface adjusted in height at the same level as the surface of the force plate. The task goal was to step forward from a stationary position and subsequently both feet came to rest next to each other (for more detail see Wang et al. 2017 [15]). The main experiment consisted of two tasks: normal stepping $(N S)$ and perturbation stepping (PS). Prior to each task, subjects performed two to three familiarization trials.

In the normal stepping task (NS), the subjects were free to initiate a forward step in a self-paced manner. In the perturbation stepping task $(P S)$, the subjects were instructed that, at any given time, the force plate would suddenly translate forward [15]. The subjects were required to react naturally to the perturbations while making a step in a self-paced manner. They were explicitly asked not to use the ankle joint to rotate the body. These instructions were given in both tasks during the experiment to ensure the same strategy was used.

Twenty trials, each $5 \mathrm{~s}$ in duration, were collected for each stepping task. Five trials of each stepping task were alternately presented to each subject. Time intervals between perturbations range from $30 \mathrm{~s}$ to $90 \mathrm{~s}$ were randomized. A minimum of 5 min seated rest was enforced after four blocks (20 trials) to prevent muscular fatigue. For safety purposes, an assistant stood in reaching distance behind the subject to prevent a fall.

\section{Data analysis}

All data were post-processed using a customized MATLAB program (R2017a, MathWorks Inc., Natick, MA, USA). Raw EMG signals were full-wave rectified and low-pass filtered (fourth-order zero-lag Butterworth filter) with a cutoff frequency of $50-\mathrm{Hz}$, while force plate signals were low-pass filtered at $20 \mathrm{~Hz}$. All trials were aligned by the toe-off time (time zero, $t_{0}$ ) using the signal from the footswitch. After alignment, rectified EMG signals were integrated over $10 \mathrm{~ms}$ intervals in a time window from -600 ms (before $t_{0}$ ) to $t_{0}$. These values were corrected by integrals of the averaged $10 \mathrm{~ms}$ baseline EMG within the $\mathrm{ST}_{\mathrm{NS}}$ condition in the time interval from $-1000 \mathrm{~ms}$ to $-900 \mathrm{~ms}$ (IEMG). $\Delta /$ IEMG indices were normalized $\left(\Delta / \mathbb{E M G}_{\mathrm{N}}\right)$ by the maximum magnitude of the integral across experimental conditions [12, 28, 29]. The timing of changes in the muscle activity (EMG onset time, $t_{\mathrm{EMG}}$ ) was defined as the instant lasting for at least $25 \mathrm{~ms}$ when the average muscle activation across trials for each condition was greater (burst) or smaller (inhibition) than the mean \pm 2 SD of its baseline activity [15]. 


\section{Defining muscle modes}

Principal component analysis (PCA) with Varimax rotation was applied to the correlation matrix of the $I E \mathrm{EG}_{\mathrm{N}}$ data from the NS condition within the time window in relation to $t_{0}$ from $-200 \mathrm{~ms}$ to $t_{0}$. The first four PCs were accepted as muscle modes (M-modes) based on the Kaiser criterion. M-modes are hypothetical neural variables manipulated by the controller to produce the $\mathrm{COP}$ shifts in the AP direction $\left(\mathrm{COP}_{\mathrm{AP}}\right)$.

\section{Defining the Jacobian matrix}

Separate multiple linear regression analysis without intercept was conducted to define the Jacobian matrix (J) for each subject. The $\mathbf{J}$ was estimated as coefficients of the multiple linear regression between small changes of $\mathrm{M}$-mode magnitudes $(\Delta \mathrm{M})$ and the changes in the $\mathrm{COP}_{\mathrm{AP}}$ shifts $\left(\triangle \mathrm{COP}_{\mathrm{AP}}\right)$.

$$
\begin{aligned}
\triangle C O P_{A P} & =k_{1} \Delta M_{1}+k_{2} \Delta M_{2}+k_{3} \Delta M_{3}+k_{4} \Delta M_{4} ; \\
J_{A P} & =\left[k_{1} k_{2} k_{3} k_{4}\right]^{\mathrm{T}}
\end{aligned}
$$

Within this approach, the $\mathbf{J}$ matrices are reduced to $(4 \times 1)$ vector-columns.

\section{Analysis of variance within the uncontrolled manifold hypothesis}

The UCM hypothesis (Scholz and Schöner 1999) allows partitioning the inter-trial variance in the M-mode space into two components [15]; the UCM $\left(\mathrm{V}_{\mathrm{UCM}}\right)$ and the space orthogonal to the UCM $\left(\mathrm{V}_{\mathrm{ORT}}\right)$. The UCM was computed as the null-space of the J matrix, where the $\mathrm{COP}_{\mathrm{AP}}$ coordinate did not change $\left(\mathrm{V}_{\mathrm{UCM}}\right)$. More details on the computational procedures are available in previously published methods [30].

An index of synergy $(\Delta V)$ was calculated:

$$
\Delta V=\frac{V_{U C M}-V_{O R T}}{V_{T O T}},
$$

where $V_{\text {TOт }}$ means the total variance and all variance indices are computed per degree of freedom. For further statistical analysis, the $\Delta V$ values were log-transformed using a Fisher's z-transformation:

$$
\Delta V_{Z}=\frac{1}{2} \cdot \log \left[\frac{4+\Delta V}{\left(1 \frac{1}{3}-\Delta V\right)}\right] .
$$

Anticipatory synergy adjustments (ASAs) prior to stepping were identified as a drop in the $\Delta V_{\mathrm{Z}}$ time profile. The time of ASA initiation $\left(t_{\mathrm{ASA}}\right)$ were grouped into two time intervals with respect to $t_{0},\{-600 ;-200\} \mathrm{ms}$
(EPAs, early postural adjustments) and $\{-200 ; 0\} \mathrm{ms}$ (APAs, anticipatory postural adjustments) [15].

\section{Statistics}

Data are presented as means \pm standard deviations (SD). We used standard Fisher's z-transformation to transform the fractions of variance explained by the first four principal components into z-scores. A paired $t$-test was conducted to compare the $\mathrm{z}$-scores and the amplitudes of the peak $\mathrm{COP}_{\mathrm{AP}}$ displacement between the conditions. Three-way ANOVA was conducted with factors Group, Condition, and Interval to compare the synergy index $\left(\Delta \mathrm{V}_{\mathrm{Z}}\right)$. Two-way ANOVAs were used with Group and Condition as the factors to compare the initiation times of $\Delta \mathrm{V}_{\mathrm{Z}}$. For all statistical tests, the level of significance was set at $p<0.05$.

\section{Results \\ General EMG patterns and COP displacements}

Figure 1 shows the normalized EMG time profiles for selected muscles, averaged across trials for representative control and dance subjects in the NS and PS conditions. Typically, the EMG pattern of the ventral and dorsal muscles showed similar time patterns and proportional activation changes in the NS condition. There was a substantial increase in the level of activity seen in most muscles of the leg/trunk under the PS condition. The subjects showed consistent patterns of muscle activation in the NS and PS conditions. Muscle activity was of a greater magnitude for the dance subjects as compared to the control subjects.

Figure 2 illustrates the averages and standard deviation values for the onsets of EMG activity for the NS and PS conditions. There was an earlier anticipatory activity of the leg and trunk muscles in the dance subjects than in the control subjects. Averaged across subjects, the onset of TA was $-118.6 \pm 45.0 \mathrm{~ms}$ to $t_{0}$ in the control group while it was $-144.7 \pm 51.8 \mathrm{~ms}$ in the dance group $\left[\mathrm{F}_{(1,14)}=4.866, p=0.045\right.$, Cohen's $\left.d=1.18\right]$. Condition significantly affected onset time in the TA and ES. Thus, the onsets of TA and ES were significantly earlier in the NS condition than in the PS condition $\left(\mathrm{F}_{(1,14)}=13.485\right.$, $p=0.003$, Cohen's $d=1.96 ; \mathrm{F}_{(1,14)}=10.433, p=0.006$, Cohen's $d=1.73$, respectively).

In the PS condition, the amplitude of the peak $\mathrm{COP}_{\mathrm{AP}}$ displacement normalized by foot length was significantly higher in the dance group than in the control group $(-22.8 \pm 2.0 \%$ vs. $-20.2 \pm 2.9 \%, p<0.05$, Cohen's $d=1.04)$. However, there were no differences between groups in the NS condition $(-9.3 \pm 2.2 \%$ vs. $-10.5 \pm 1.9 \%, p>0.05$, Cohen's $d=0.58$ ). Note that the negative values correspond to backward displacements. 


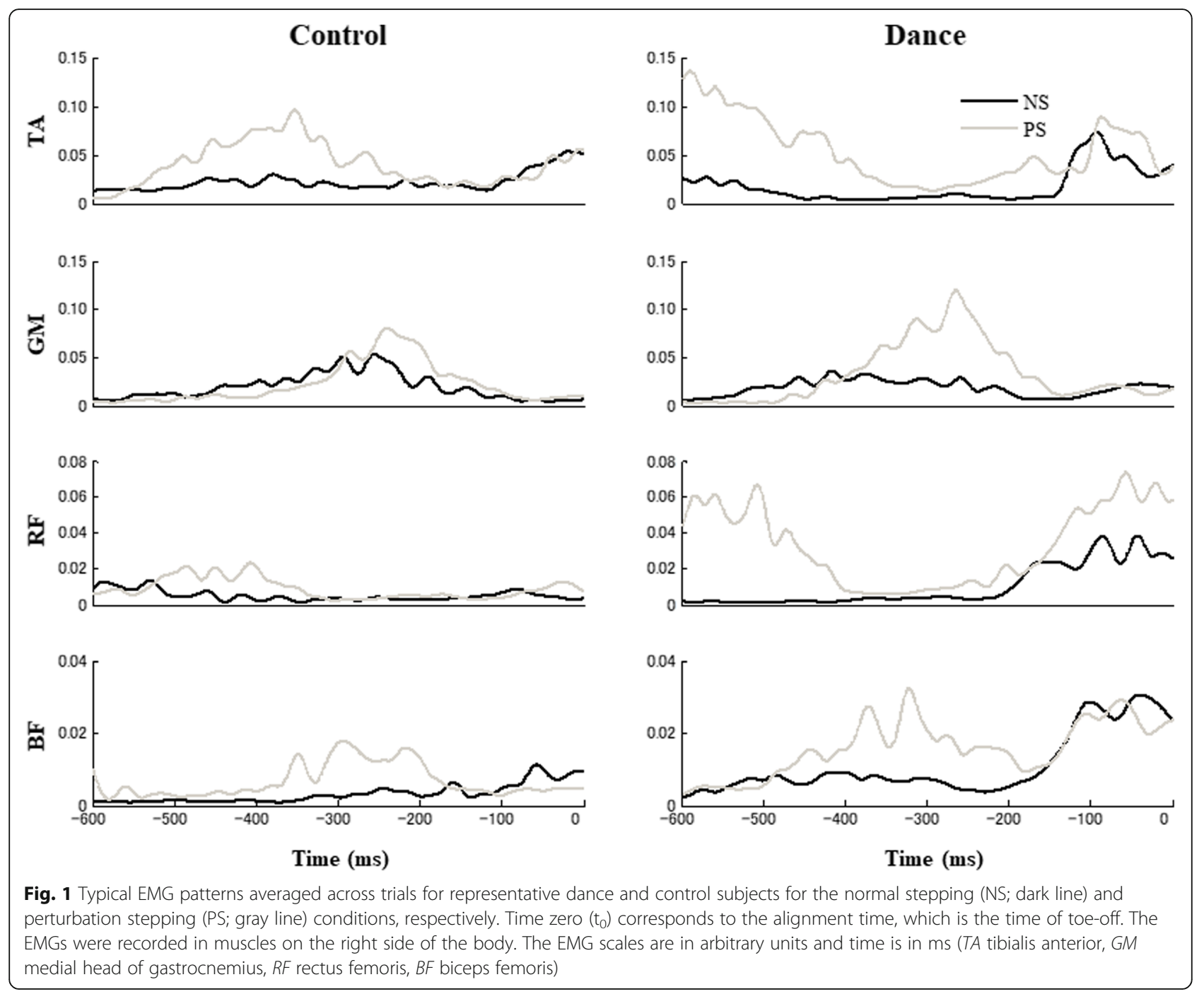

\section{PCA and multiple regression analysis}

M-modes were identified using principal component analysis with Varimax rotation to identify muscle groups (eigenvectors in the muscle activation space) using the normalized integrated EMG indices under the NS condition. M-modes represent unitary vectors in the muscle activation space that can be recruited by the controller with different magnitudes. Four M-modes were identified in all subjects. On average, the first four principal components (which we refer to as muscle modes, $M_{1}$, $\mathrm{M}_{2}, \mathrm{M}_{3}$, and $\mathrm{M}_{4}$ ) accounted for about $62.4 \pm 5.7 \%$ of the total variance in the muscle activation space in the dance group and $62.2 \pm 4.2 \%$ in the control group. Table 2 presents the across-subjects average amount of variance explained by each M-mode. In the dance group, the average amount of variance explained by PC1 was $20.1 \pm 2.7 \%$, by PC2 was $16.8 \pm 3.4 \%$, by PC3 was $13.8 \pm$ $1.3 \%$ and by PC4 was $11.7 \pm 1.2 \%$. Similarly, in the control group, the average amount of variance explained by
PC1 was $20.9 \pm 2.4 \%$, by PC2 was $16.2 \pm 2.6 \%$, by PC3 was $13.5 \pm 1.9 \%$ and by PC4 was $11.6 \pm 1.3 \%$. For statistical analysis, variance values were transformed into z-scores. One-way ANOVA with the factor Group was used separately for each PC data. The ANOVA revealed no significant differences between groups.

Individual loadings for all the muscles for a representative dance subject are presented in Fig. 3 (bottom panel). The significant loadings are shown in gray. The first M-mode showed high loading values with the same sign for the IEMG indices of the dorsal muscles (dorsal M-mode), while the second M-mode showed high loading values for the IEMG indices of the ventral muscles, also with the same sign (ventral M-mode). The third Mmode again was dorsal M-mode. The fourth M-mode showed high loading values with the same sign for the IEMG indices for the muscles acting at the trunk (mixed M-mode). There was considerable variability across the subjects in the M-mode composition. 


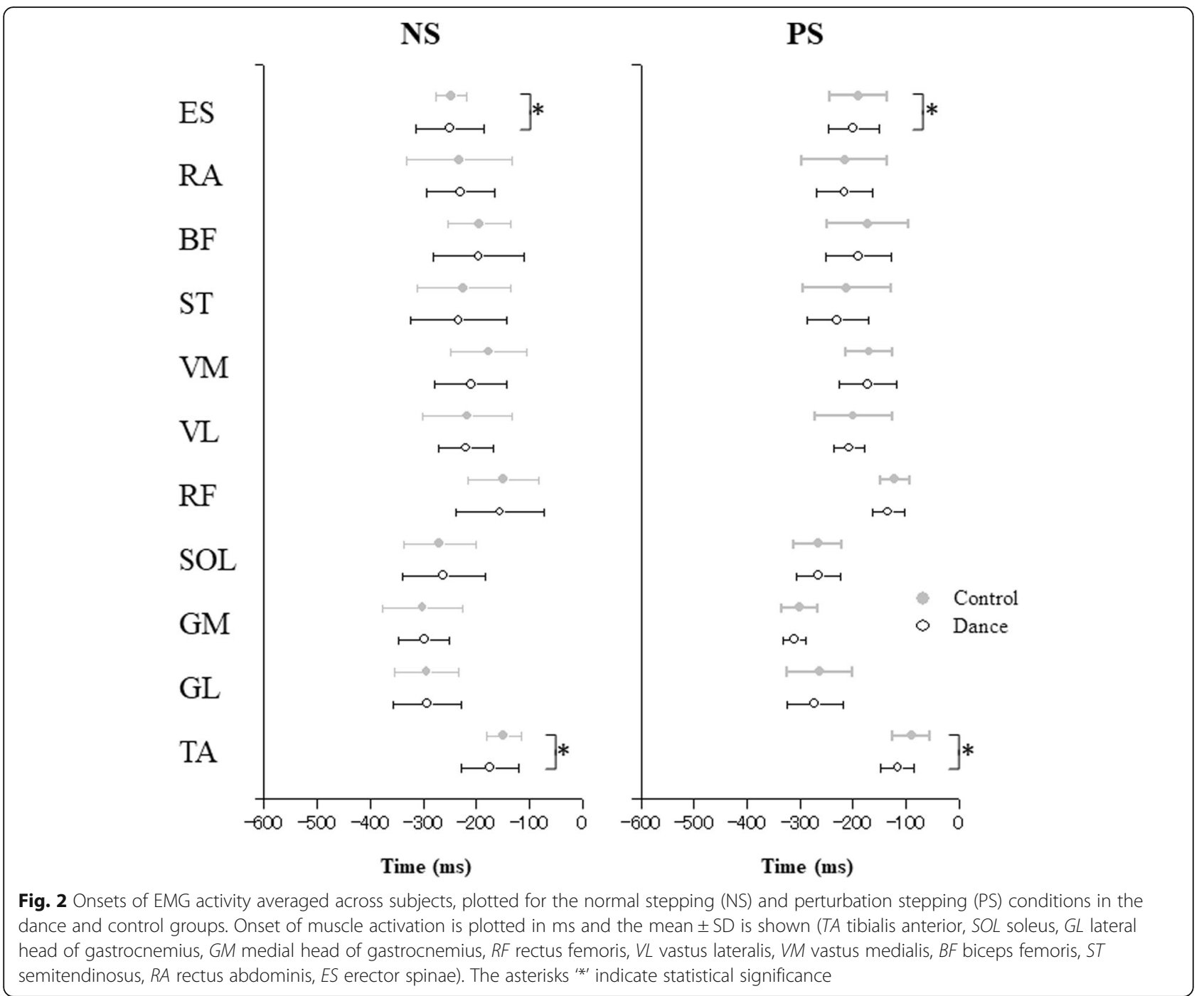

For each subject, the number of times that dorsal Mmodes, ventral $\mathrm{M}$-modes, and mixed $\mathrm{M}$-modes were seen under the $\mathrm{ST}_{\mathrm{NS}}$ condition was summed (Table 3). Overall, in the dance group, $10 \mathrm{M}$-modes with mixed Mmodes was seen, while in the control group, the number of mixed M-modes was 14 (from a total of $36 \mathrm{M}$-modes). The number of times the mixed M-modes was seen in the control group was significantly larger than that of the dance group $(\mathrm{U}=15.0, p=0.042, r=-0.508)$.

Multiple regression analysis was used to define the Jacobian mapping small changes in the magnitude of M-modes $(\Delta \mathrm{M})$ against the change of $\mathrm{COP}_{\mathrm{AP}}$ shifts $\left(\Delta \mathrm{COP}_{\mathrm{AP}}\right)$. In most cases, $\Delta \mathrm{M}$ values were significant predictors of $\triangle \mathrm{COP}_{\mathrm{AP}}$ for each of the two groups. On average, the analysis accounted similar for $85.7 \pm 3.8 \%$ and $84.3 \pm 4.4 \%$ of variance in $\triangle \mathrm{COP}_{\mathrm{AP}}$ in the dance and control groups, respectively (no group difference).

\section{Analysis of M-mode variance}

We analyzed M-mode variance using the framework of the UCM hypothesis to investigate whether M-modes co-varied across trials to stabilize the $\mathrm{COP}_{\mathrm{AP}}$ shift prior to stepping. A three-way Group $\times$ Condition $\times$ Interval ANOVA was performed to analyze the effect of dance on $\mathrm{M}$-mode synergies $\left(\Delta \mathrm{V}_{\mathrm{Z}}\right)$. The $\Delta \mathrm{V}_{\mathrm{Z}}$ values computed for the $\mathrm{COP}_{\mathrm{AP}}$ shift as the performance variable became

Table 2 Total variance explained by the first four principal components

\begin{tabular}{|c|c|c|c|c|}
\hline Group & PC1 (M M $_{1}$ mode $)$ & PC2 (M -mode) & PC3 (M & PC4 (M4-mode) \\
\hline Control & $20.9 \pm 2.4 \%$ & $16.2 \pm 2.6 \%$ & $13.5 \pm 1.9 \%$ & $11.6 \pm 1.3 \%$ \\
\hline Dance & $20.1 \pm 2.7 \%$ & $16.8 \pm 3.4 \%$ & $13.8 \pm 1.3 \%$ & $11.7 \pm 1.2 \%$ \\
\hline
\end{tabular}




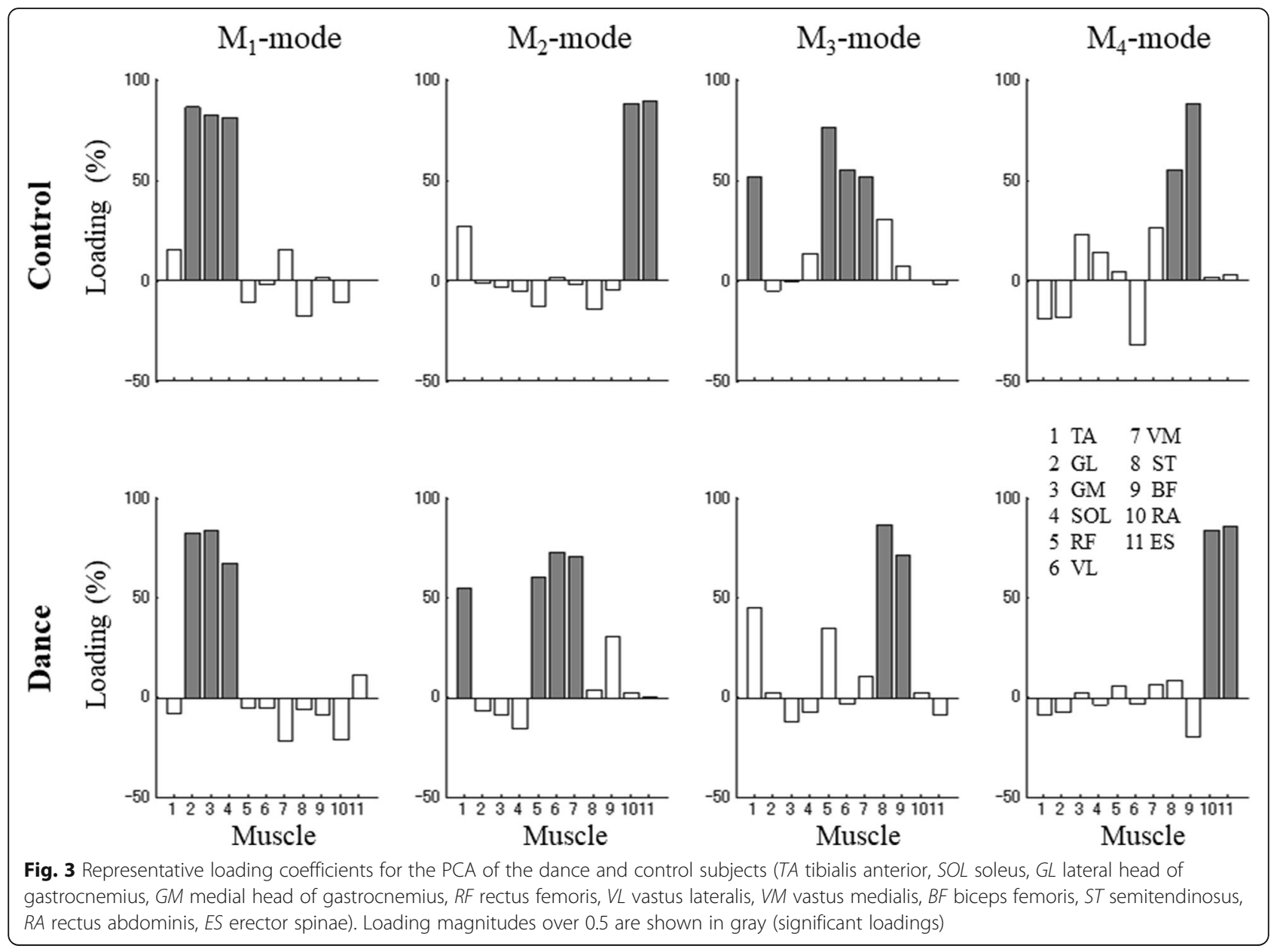

greater for the dance subjects $\left(\Delta \mathrm{V}_{\mathrm{Z}}=1.16 \pm 0.47\right)$ than for the control subjects $\left(\Delta V_{Z}=0.91 \pm 0.50\right)$, and the trend of $\Delta \mathrm{V}_{\mathrm{Z}}$ across time was similar for both groups. The difference between the groups was significant. This finding was confirmed by the significant effect of Group $\left[\mathrm{F}_{(1,14)}=5.235, p=0.038\right.$, Cohen's $\left.f=0.28\right]$ on $\Delta \mathrm{V}_{\mathrm{Z}}$. Additionally, the main effect of Condition $\left[\mathrm{F}_{(1,70)}=6.851\right.$, $p=0.020$, Cohen's $f=0.35]$ showed that $\Delta \mathrm{V}_{\mathrm{Z}}$ in the $\mathrm{ST}_{\mathrm{NS}}$ condition had significantly higher values compared to the $\mathrm{ST}_{\mathrm{PS}}$ condition. There were none of the time effects or interactions were significant.

Figure 4 shows time profiles of the synergy index, $\Delta V_{Z}$ computed for the NS and PS conditions in representative dance and control subjects. $\Delta \mathrm{V}_{\mathrm{Z}}$ was qualified over six 100 -ms time intervals from $-600 \mathrm{~ms}$ prior to $t_{0}$ to $t_{0}$. The two $\Delta \mathrm{V}_{\mathrm{Z}}$ dropped from positive initial values during the EPA time interval. There was also a substantial drop during the APA time interval. Figure 5 shows the across-subjects average time of the $\Delta \mathrm{V}_{\mathrm{Z}}$ drop for the EPA and APA time intervals. Two-way Group $\times$ Condition ANOVA on the timing indices for the EPA interval showed a main effect of Group $\left[\mathrm{F}_{(1,14)}=6.735, p=0.021\right.$, Cohen's $f=0.34]$. Compared to the control group, the timing indices were significantly earlier in the dance group. For the APA time interval, $\Delta \mathrm{V}_{\mathrm{Z}}$ dropped significantly earlier in the NS condition than in the PS condition. Two-way Group $\times$ Condition ANOVA confirmed a significant effect of Condition $\left[\mathrm{F}_{(1,14)}=7.708, p=0.015\right.$, Cohen's $f=0.37$ ] without other significant Condition effect or interaction.

\section{Discussion}

Here we show 5-years of dance in older adults is associated with changes in multi-muscle postural synergies stabilizing the COP displacement during the preparation for making a step associated with support surface translations. We investigated dance induced changes in the synergy index $\left(\Delta \mathrm{V}_{\mathrm{Z}}\right)$, computed as the normalized difference between $V_{U C M}$ and $V_{O R T}$ based on the uncontrolled manifold hypothesis: $\mathrm{V}_{\mathrm{UCM}}$ that had no effect on the COP coordinate and $\mathrm{V}_{\mathrm{ORT}}$ that affected the COP coordinate. We demonstrate that 5-years of dance altered the composition of muscle groups and synergies stabilizing the COP coordinate for making a step and balance. Our results, together with the results of an earlier study, which documented synergy index and ASA differences 
Table 3 The number of occurrences of different M-modes

\begin{tabular}{|c|c|c|}
\hline \multirow[t]{2}{*}{ M-modes Pattern } & \multicolumn{2}{|l|}{ Group } \\
\hline & Control & Dance \\
\hline Dorsal M-modes & 11 & 14 \\
\hline Ventral M-modes & 7 & 8 \\
\hline Mixed M-modes & 14 & $10^{*}$ \\
\hline Hip co-contraction & 5 & 6 \\
\hline Knee co-contraction & 5 & 2 \\
\hline Ankle co-contraction & 0 & 0 \\
\hline Hip-dorsal & 0 & 0 \\
\hline Hip-ventral & 1 & 1 \\
\hline Ankle-dorsal & 0 & 0 \\
\hline Ankle-ventral & 0 & 0 \\
\hline Knee-dorsal & 0 & 0 \\
\hline Singular & 3 & 1 \\
\hline $\begin{array}{l}{ }^{*} p<0.05 \\
\text { Data across all subjects are } \\
\text { Hip-dorsal: the combinatior } \\
\text { dorsal M-mode } \\
\text { Hip-ventral: the combinatio } \\
\text { ventral M-mode } \\
\text { Ankle-dorsal: the combinat } \\
\text { SOL) and dorsal M-mode } \\
\text { Ankle-ventral: the combina } \\
G M+S O L) \text { and "ventral M-r } \\
\text { Knee-dorsal: the combinati } \\
\text { BF + ST, GM } \mathrm{VL} \text { ) and dors } \\
\text { Singular: only one muscle I } \\
\text { The muscles indicated in it } \\
\text { contraction indicated. TA ti } \\
\text { gastrocnemius, GM medial } \\
\text { lateralis, } V M \text { vastus medialis } \\
\text { abdominis, ES erector spina }\end{array}$ & $\begin{array}{l}\text { ction at the } \\
\text { tly on one } \\
\text { W up cons } \\
O L \text { soleus, } \\
\text { nemius, } R F \\
\text { oris, ST sen }\end{array}$ & $\begin{array}{l}d \\
+G M+ \\
\mathrm{d}+ \\
V M+ \\
\text { Cs } \\
L \text { vastus } \\
\text { ectus }\end{array}$ \\
\hline
\end{tabular}

between older adults and young controls [13], point at important implications for a variety of issues related to synergic control of muscles in postural tasks.

Since Bernstein [31], it has been recognized that postural synergies play a fundamental role in the control and stabilization of posture and movement. Features of muscle coordination during gait and while changing from one posture to another may provide important insight into neurophysiological mechanisms of synergic control of postural stability and motor performance. Muscle synergy analysis may thus provide such insight and identify differences in neuromuscular coordination of multi-muscle whole-body actions [32]. Recent progress in the analysis of apparently redundant systems has led to an operational definition of multi-muscle synergies, and a method to quantify synergies within the UCM hypothesis $[15,33]$. Based on this framework, Mmodes are defined as elemental variables to control vertical posture manipulated by the CNS [32-34]. Mmodes are groups of coactive muscles with proportional scaling of muscle activation levels. For the individual muscle activation space, typical sets of $\mathrm{M}$-modes are low-dimensional. However, they are still redundant as compared to important performance variables [35-37]. The UCM hypothesis assumes that the CNS is able to manipulate gains at the M-modes space to ensure that most M-mode variance lies within the UCM where the salient performance variable(s) did not change. The UCM approach views the multi-muscle postural synergies as involving a two-level hierarchy. At the lower hierarchical level, muscles are organized into M-modes, while at the higher hierarchical level magnitudes of M-modes co-vary to produce required action.

Our work is the first to show the effectiveness of dance on muscle synergies in older adults. In both groups, the first four principal components (M-modes) represented linear combinations of activation for eleven leg and trunk muscles. The dance group had more reciprocal M-modes (with significantly loaded ventral or dorsal muscles) than the control group. There were only a few cases of co-contraction M-modes (those involving parallel changes in the activation levels of agonistantagonist muscles acting at leg/trunk joints) in the dance group despite the fact that the control group showed similar variances were accounted for by the four M-modes. This result suggests that both subject groups used a consistent set of M-modes over the performance of the stepping task; however, the dance could lead to a transition from mixed muscle activation patterns to reciprocal patterns and as a consequence to improve overall balance control. Our results are consistent with the previous study on balance training in older adults [38]. Studies of different postural tasks have shown that M-modes correspond to the COP displacement may be critical to the control of posture and movement, during voluntary, whole-body tasks $[39,40]$. The reciprocal Mmodes may effectively move the center of mass, while the mixed M-modes may increase the apparent joint stiffness. Our finding of significant changes in the Mmode composition is quite similar to the earlier report on the more common occurrence of reciprocal $\mathrm{M}$ modes with practice [30]. But their study observed the healthy young subjects that differ somewhat from ours. M-modes can scale and recombine with significant changes associated with dance. This result fits the notion of the earlier works. Since the muscle activities are dependent on each other $[24,25]$ and redundant, the neural controller forms flexible M-modes by combining the individual muscles to stabilize the COP coordinate. Our finding shows that the effects of dance on muscle synergies may involve changes at different levels of the hierarchical control system to meet the task requirements. This result allows us to hope that dance can lead to improved synergic control of postural stability in older adults.

We have previously shown that the index of M-mode synergies in older adults was significantly lower and 


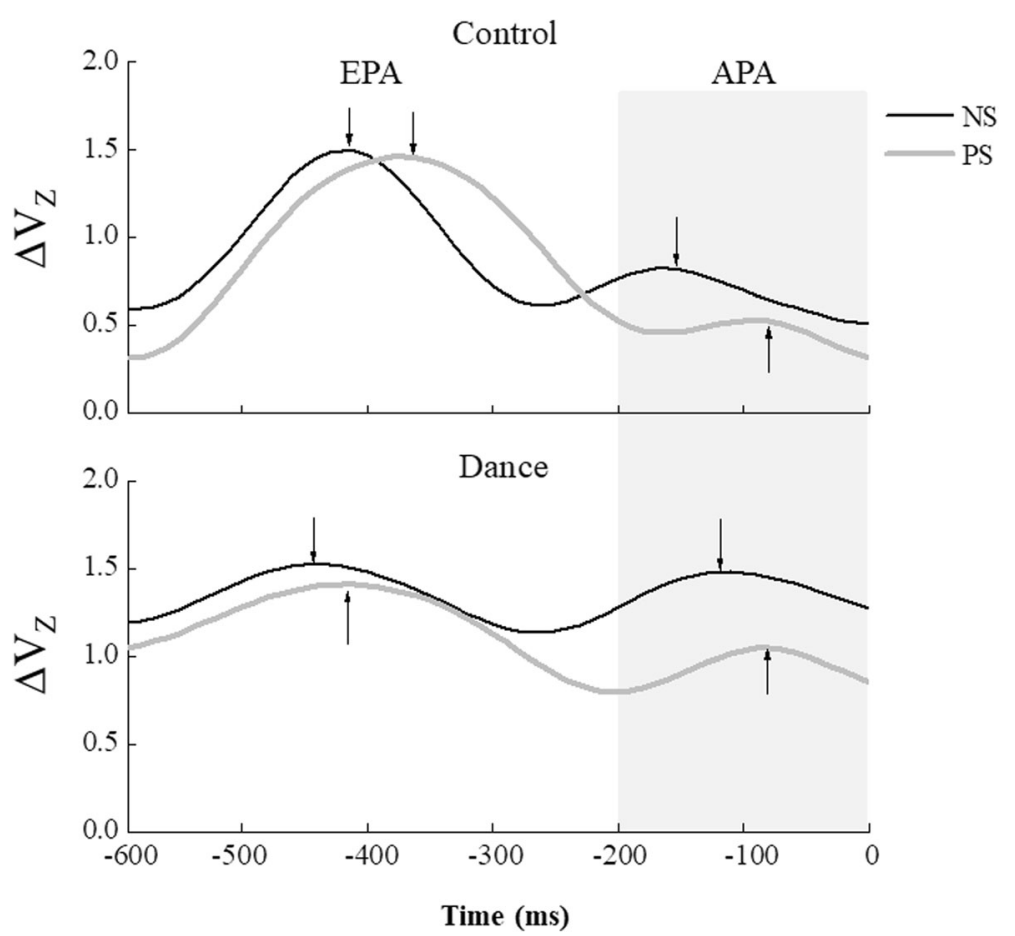

Fig. 4 Time profiles of the $\Delta V_{z}$ index for representative dance and control subjects in the normal stepping (NS; dark line) and perturbation stepping (PS; gray line) conditions. The two $\Delta V_{Z}$ profiles started with positive values and included a transient drop in $\Delta V_{z}$ during the early postural adjustment (EPA) time interval followed by another drop during the anticipatory postural adjustment (APA) time interval (gray-shaded area).The arrows represent drops of anticipatory synergy adjustment (ASA). Time zero ( $\mathrm{t}_{0}$ ) corresponds to the alignment time, which is the time of toe-off

delayed as compared to the young subjects during preparation for making a step associated with support surface translation [27]. In the current study, these synergy index values, on average, were significantly higher in the dance group than in the control group. Additionally, the timings of EPAs and APAs were consistently earlier in dance subjects as compared to the control subjects. This may be interpreted as an improvement in the ability to organize $\mathrm{M}$-modes into $\mathrm{COP}_{\mathrm{AP}}$ stabilizing synergies in the dance group.

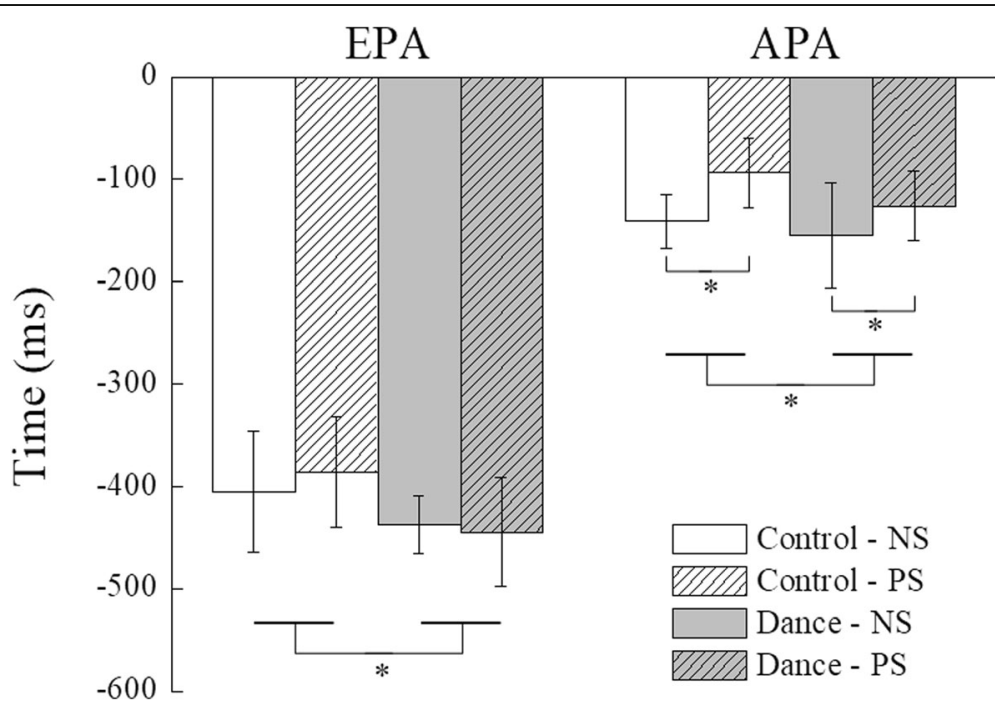

Fig. 5 Timing indices for the early postural adjustment (EPA) and anticipatory postural adjustment (APA) averaged across subjects are shown for the normal stepping (NS) and perturbation stepping (PS) conditions for the dance and control groups. The asterisks '*' indicate statistical significance 
In preparation for a whole-body postural task, ASAs facilitate the destabilization of the COP coordinate by making it less stable $[27,39,40]$. When a standing person initiates a step, there are relatively quick COP shifts with the purpose to unload the stepping leg and to generate a moment of the vertical force rotating the body forward about the ankle joints. These COP shifts are mechanically necessary to make a step. If the COP coordinate is stabilized by a strong M-mode synergy [32, 41], the person may be unable to produce COP shifts unless ASAs attenuate the synergy. Indeed, ASAs modify preexistent synergies prior to the classical APAs to ensure that the body does not have to fight its own synergies stabilizing the COP coordinate to be changed.

Delayed and reduced ASAs could potentially cause age-related impairments in synergic control [11, 42]. Several studies have shown that impaired control of postural stability happens with healthy aging $[12,13,43]$. In those studies, older adults showed a reduction in the synergy index in whole-body postural tasks in preparation for action. There was a delay in the onset latencies of ASAs and more time needed to stabilize the COP coordinate. In the current study, the dance group showed significant changes in the synergy indexes and the timing of ASAs with dance. This observation corroborates the idea of dance leading to more reproducible performance and supports using the synergy index to assess such changes.

Limitations of this study include its cross-sectional design with the single time point comparative analysis, the relatively small sample size and lack of information about lower limb muscle strength. The stringent inclusion criteria have hindered our ability to recruit more subjects, which may have affected the data and limit conclusions. The strength of this study design is that each subject completed 20 trials in each task, and thus the precision of the results would be strong. Whereas recent study has shown dance doesn't improve muscle strength [44], we cannot exclude the possible contributions of muscle strength in the current experiment. In follow-ups to this study, we shall explore to greater depth how muscle strength is altered by dance invention to multi-muscle synergies.

\section{Conclusions}

To the authors' knowledge, this is the first demonstration of dance leads to more reciprocal muscle activation patterns that stabilized COP displacement to forward perturbation of the support surface in older adults. Additionally, dance was associated with higher indices of multi-muscle synergies and earlier ASAs timing. The findings of this cross-sectional study, albeit preliminary, suggest that the effects of dance on motor coordination may involve the M-mode composition and M-mode co-variation patterns for control and maintenance of postural stability of multi-muscle action. Recent analyses of postural stability in multi-muscle tasks in neurological patients have shown its high sensitivity to several neurological disorders [32, 45]. Quantifying multi-muscle synergies during balance could potentially inform improved rehabilitative outcome measures. Therefore, the results reported here could have clinical relevance when offering a dance approach to balance training for impaired individuals.

\section{Abbreviations}

AP: Anterior-posterior; APAs: Anticipatory postural adjustments; ASAs: Anticipatory synergy adjustments; BF: Biceps femoris; COP: Center of pressure; EPAs: Early postural adjustments; ES: Erector spinae; GL: Lateral head of gastrocnemius; GM: Medial head of gastrocnemius; PCA: Principal component analysis; RA: Rectus abdominis; RF: Rectus femoris; SOL: Soleus; ST: Semitendinosus; TA: Tibialis anterior; UCM: Uncontrolled manifold; VL: Vastus lateralis; VM: Vastus medialis

\section{Acknowledgements}

The authors would like to thank Xifang Cui's dance class for participating in this study.

\section{Authors' contributions}

YW conceived of the study, participated in its design and setup of the study, performed data collection, preparations and statistical analyses, and drafted the manuscript. KW participated in the design, setup and coordination of the study. TA participated in the results interpretation. All authors read and approved the final manuscript.

\section{Funding \\ This work was supported by the National Natural Science Foundation of China [grant \#31371207] and the Natural Science Foundation of Tianjin [grant \#14JCYBJC43300]. The funding bodies did not play any role in the design of the study and collection, analysis, and interpretation of data and in writing the manuscript.}

\section{Availability of data and materials}

The datasets used and/or analyzed in the presented study are available from the corresponding author on reasonable request.

\section{Ethics approval and consent to participate}

The study was approved by the Tianjin University of Sport's Institutional Review Board [IRB \#13212] and all methods were carried out in accordance with the approved study protocol. The subjects provided written informed consent before participation.

Consent for publication Not applicable.

\section{Competing interests}

The authors declare that they have no competing interests.

\section{Author details}

${ }^{1}$ Tianjin Key Lab of Exercise Physiology and Sports Medicine, College of Social Sport and Health Sciences, Tianjin University of Sport, 16 Donghai Road, Tuanbo Xincheng Xiqu, Jinghai District, Tianjin 301617, China. ${ }^{2}$ Institute of Sports and Health Science, 3-10-31, Kagamiyama, Higashi-hiroshima, Hiroshima 739-0046, Japan. ${ }^{3}$ Department of Rehabilitation Science, Faculty of Health Sciences, Hokkaido University, N12-W5, Kita-ku, Sapporo 060-0826, Japan. 
Received: 21 November 2018 Accepted: 22 November 2019

Published online: 03 December 2019

\section{References}

1. Crenna P, Frigo C. A motor programme for the initiation of forwardoriented movements in humans. J Physiol. 1991;437:635-53.

2. Winter DA, Mackinnon CD, Ruder GK, Wieman C. An integrated EMG/ biomechanical model of upper balance and posture during human gait. Prog Brain Res. 1993;97:359-67.

3. Jensen $J$, Brown LA, Woollacott MH. Compensatory stepping: the biomechanics of a preferred response among older adults. Exp Ageing Res. 2001;27:361-76.

4. Mille $M L$, Rogers MW, Martinez $K$, et al. Thresholds for inducing protective stepping responses to external perturbations of human standing. J Neurophysiol. 2003;90:666-74.

5. Vaillancourt DE, Larsson L, Newell KM. Effects of aging on force variability, motor unit discharge patterns, and the structure of 10,20, $40 \mathrm{~Hz}$ EMG activity. Neurobiol Aging. 2003;24:25-35.

6. Lin SI, Woollacott MH, Jensen JL. Postural response in older adults with different levels of functional balance capacity. Aging Clin Exp Res. 2004;16:369-74

7. Melzer I, Benjuya N, Kaplanski J. Postural stability in the elderly: a comparison between fallers and non-fallers. Age Aging. 2004;33:602-7.

8. Klous M, Mikulic P, Latash ML. Two aspects of feed-forward postural control: anticipatory postural adjustments and anticipatory synergy adjustments. J Neurophysiol. 2011;105(5):2275-88.

9. Cordo PJ, Nashner LM. Properties of postural adjustments associated with rapid arm movements. J Neurophysiol. 1982;47:1888-905.

10. Horak FB. Postural orientation and equilibrium: what do we need to know about neural control of balance to prevent falls? Age Ageing. 2006;35(Suppl 2):ii7-ii1 1

11. Olafsdottir HB, Yoshida N, Zatsiorsky VM, Latash ML. Elderly show decreased adjustments of motor synergies in preparation to action. Clin Biomech. 2007;22:44-51

12. Wang $Y$, Watanabe $K$, Asaka T. Muscle synergies in preparation to a step made with obstacle in elderly individuals. J Neuroeng Rehabil. 2015;12:10.

13. Wang $Y$, Watanabe $K$, Asaka T. Aging effect on muscle synergies in stepping forth during a forward perturbation. Eur J Appl Physiol. 2017;117(1):201-11.

14. Gelfand IM, Latash ML. On the problem of adequate language in motor control. Mot Control. 1998;2(4):306-13.

15. Scholz JP, Schöner G. The uncontrolled manifold concept: identifying control variables for a functional task. Exp Brain Res. 1999;126:289-306.

16. Latash ML. Motor synergies and the equilibrium-point hypothesis. Mot Control. 2010;14(3):294-322.

17. Vaz DV, Pinto VA, Junior RRS, Mattos DJS, Mitra S. Coordination in adults with neurological impairment - a systematic review of uncontrolled manifold studies. Gait Posture. 2019;69:66-78.

18. Anan M, Hattori H, Tanimoto K, Wakimoto Y, Ibara T, Kito N, Shinkoda K. The coordination of joint movements during sit-to-stand motion in old adults: the uncontrolled manifold analysis. Phys Ther Res. 2017:20(2):44-50.

19. Olafsdottir HB, Zatsiorsky VM, Latash ML. The effects of strength training on finger strength and hand dexterity in healthy elderly individuals. J Appl Physiol. 2008;105:1166-78.

20. Wu YH, Pazin N, Zatsiorsky VM, Latash ML. Improving finger coordination in young and elderly persons. Exp Brain Res. 2013;226:273-83.

21. Verghese J. Cognitive and mobility profile of older social dancers. J Am Geriatr Soc. 2006;54(8):1241-4.

22. Zhang JG, Ishikawa-Takata K, Yamazaki H, Morita T, Ohta T. Postural stability and physical performance in social dancers. Gait Posture. 2008;27:697-701.

23. Hackney M, Kantorovich S, Earhart GM. A study on the effects of argentine tango as a form of partnered dance for those with Parkinson disease and the healthy elderly. Am J Dance Ther. 2007;29(2):109-27.

24. Hughlings J. On the comparative study of disease of the nervous system. $\mathrm{Br}$ Med J. 1889;17:355-62

25. Lee WA. Neuromotor synergies as a basis for coordinated intentional action. J Mot Behav. 1984;16:135-70

26. Nachshon I, Denno D, Aurand S. Lateral preference of hand, eye and foot: relation to cerebral dominance. Int J Neurosci. 1983;18(1-2):1-9.

27. Wang Y, Watanabe $K$, Asaka T. Muscle synergies underlying control of taking a step during support surface translation. Eur J Appl Physiol. 2016;116(2):301-9.
28. Asaka T, Wang Y. Feedforward postural muscle modes and multi-mode coordination in mild cerebellar ataxia. Exp Brain Res. 2011:210:153-63.

29. Wang Y, Zatsiorsky VM, Latash ML. Muscle synergies involved in shifting the center of pressure while making a first step. Exp Brain Res. 2005;167(2):196-210.

30. Asaka T, Wang Y, Fukushima J, Latash ML. Learning effects on muscle modes and multi-mode postural synergies. Exp Brain Res. 2005;184(3):323-38.

31. Latash ML. Muscle coactivation: definitions, mechanisms, and functions. J Neurophysiol. 2018;120(1):88-104

32. Krishnamoorthy V, Goodman S, Zatsiorsky V, Latash ML. Muscle synergies during shifts of the center of pressure by standing persons: identification of muscle modes. Biol Cybern. 2003;89:152-61.

33. Danna-Dos-Santos A, Slomka K, Zatsiorsky VM, Latash ML. Muscle modes and synergies during voluntary body sway. Exp Brain Res. 2007:179:533-50.

34. Wang Y, Asaka T. Muscle synergies involved in shifts of the center of pressure while standing on a narrow support. Brain Res Bull. 2008;76(1-2):16-25

35. Robert T, Zatsiorsky VM, Latash ML. Multi-muscle synergies in an unusual postural task: quick shear force production. Exp Brain Res. 2008;187(2):237-53.

36. Klous M, Danna-dos-Santos A, Latash ML. Multi-muscle synergies in a dual postural task: evidence for the principle of superposition. Exp Brain Res. 2010;202:457-71.

37. Nagai K, Yamada M, Tanaka B, Uemura K, Mori S, Aoyama T, et al. Effects of balance training on muscle coactivation during postural control in older adults: a randomized controlled trial. J Gerontol A Biol Sci Med Sci. 2012;67(8):882-9.

38. Piscitelli D, Falaki A, Solnik S, Latash M. Anticipatory postural adjustments and anticipatory synergy adjustments: preparing to a postural perturbation with predictable and unpredictable direction. Exp Brain Res. 2017;235:713-30.

39. Nardini A, Freitas S, Falaki A, Latash M. Preparation to a quick whole-body action: control with referent body orientation and multi-muscle synergies. Exp Brain Res. 2019;237:1361-74.

40. Wang Y, Zatsiorsky VM, Latash ML. Muscle synergies involved in preparation to a step made under the self-paced and reaction time instructions. Clin Neurophysiol. 2006;117:41-56.

41. Kapur S, Zatsiorsky VM, Latash ML. Age-related changes in the control of finger force vectors. J Appl Physiol. 2010;109:1827-41.

42. Wang Y, Asaka T, Watanabe K. Multi-muscle synergies in elderly individuals: preparation to a step made under the self-paced and reaction time instructions. Exp Brain Res. 2013;226(4):463-72.

43. Serra MM, Alonso AC, Peterson M, Mochizuki L, Greve JM, Garcez-Leme LE. Balance and muscle strength in elderly women who dance samba. PLoS One 2016:11(12):e0166105

44. Latash ML, Huang X. Neural control of movement stability: lessons from studies of neurological patients. Neurosci. 2015;301:39-48.

45. Bernstein N. The coordination and regulation of movements. London: Pergamon Press; 1967.

\section{Publisher's Note}

Springer Nature remains neutral with regard to jurisdictional claims in published maps and institutional affiliations.

Ready to submit your research? Choose BMC and benefit from:

- fast, convenient online submission

- thorough peer review by experienced researchers in your field

- rapid publication on acceptance

- support for research data, including large and complex data types

- gold Open Access which fosters wider collaboration and increased citations

- maximum visibility for your research: over $100 \mathrm{M}$ website views per year

At BMC, research is always in progress.

Learn more biomedcentral.com/submission 